\title{
Homotopy Analysis Method for Solving Fuzzy Integro-Differential Equations
}

\author{
Eman A. Hussain ${ }^{1} \&$ Ayad W. Ali ${ }^{1}$ \\ ${ }^{1}$ Department of Mathematics, College of Science, AL-Mustansiriyah University, Baghdad, Iraq \\ Correspondence: Eman A. Hussain, Department of Mathematics, College of Science, AL-Mustansiriyah \\ University, Baghdad, Iraq. E-mail: dr_emansultan@yahoo.com
}

Received: January 20, 2013

Accepted: February 15, 2013 Online Published: February 22, 2013

doi:10.5539/mas.v7n3p15

URL: http://dx.doi.org/10.5539/mas.v7n3p15

\begin{abstract}
In this paper, the numerical solution of the linear and nonlinear fuzzy Volterraintegro-differential equations have been investigated using the analytical method namely homotopy analysis method (HAM) and then the proposed method is illustrated by solving two numerical examples. It was found that the HAM provides a simple way to adjust and control the convergence region of solution series by introducing a nonzero auxiliary parameter $\hbar$.
\end{abstract}

Keywords: fuzzy numbers, fuzzy volterraintegro-differential equations, homotopy analysis method

\section{Introduction}

Integro-differential equations (IDEs) play an important role in many branches of linear and nonlinear functional analysis and their applications in the theory of engineering, mechanics, physics, chemistry, astronomy, biology, economics, potential theory and electrostatics. The fuzzy functions were introduced by Chang and Zadeh (1972). Later the concept of integration of fuzzy functions was introduced by Dobois and Prade (1982). In 1992, Liao employed the basic ideas of the homotopy, a fundamental concept in topology and differential geometry, to propose a general analytic method for linear and nonlinear problems, called Homotopy Analysis Method (HAM) (Liao, 2003; 2004; 2009 a \& b). The homotopy analysis method (HAM) is a general analytic approach to get series solutions of various types of nonlinear equations, including algebraic equations, ordinary differential equations, partial differential equations, differential-integral equations, differential-difference equation and coupled equations of them. This method has been successfully applied to solve many types of linear and nonlinear problems (Afroozi, Vahidi, \& Saeidy, 2010; Abbasbandy, Magyari, \& Shivanian, 2009; Abbasbandy, Babolian, \& Ashtiani, 2009; Bataineh, Noorani, \& Hashim, 2008; Dubois \& Prade, 1982; Ghanbari, 2010; Liao \& Tan, 2007).

\section{Preliminaries}

In this section, the most basic notations used in fuzzy calculus are introduced. We start with defining a fuzzy number.

Definition 2.1 (Goetschel \& Voxman, 1983) A fuzzy number is a map u: $\mathrm{R} \rightarrow \mathrm{I}=[0,1]$ which satisfies

i. $\mathrm{u}$ is upper semi-continuous,

ii. $\mathrm{u}(\mathrm{x})=0$ outside some interval $[\mathrm{c}, \mathrm{d}]$,

iii. There exist real numbers $\mathrm{a}, \mathrm{b}$ such that $\mathrm{c} \leq \mathrm{a} \leq \mathrm{b} \leq \mathrm{d}$, where

1) $u(x)$ is monotonic increasing on $[c, a]$,

2) $u(x)$ is monotonic decreasing on $[b, d]$,

3) $u(x)=1, \quad a \leq x \leq b$.

An equivalent parametric definition of fuzzy numbers is given by Friedman, Ma, and Kandel (1999) as follows:

Definition 2.2 An arbitrary fuzzy number in parametric form is represented by an ordered pair of functions $(\underline{\mathrm{u}}(\alpha), \overline{\mathrm{u}}(\alpha)) \quad 0 \leq \alpha \leq 1$, which satisfying the following requirements:

i. $\underline{\mathrm{u}}(\alpha)$ is a bounded left-continuous non-decreasing function over $[0,1]$,

ii. $\overline{\mathrm{u}}(\alpha)$ is a bounded left-continuous non-increasing function over $[0,1]$, 
iii. $\underline{\mathrm{u}}(\alpha) \leq \overline{\mathrm{u}}(\alpha), \quad 0 \leq \alpha \leq 1$.

For arbitrary fuzzy numbers $\mathrm{u}=(\underline{\mathrm{u}}(\alpha), \overline{\mathrm{u}}(\alpha)), \mathrm{v}=(\underline{\mathrm{v}}(\alpha), \overline{\mathrm{v}}(\alpha))$ and real constant we define addition $\mathrm{u}+\mathrm{v}$ and scalar multiplication $\mathrm{ku}$ as

$$
\begin{aligned}
& (\underline{\mathrm{u}+\mathrm{v}})(\alpha)=\underline{\mathrm{u}}(\alpha)+\underline{\mathrm{v}}(\alpha), \\
& (\overline{\mathrm{u}+\mathrm{v}})(\alpha)=\overline{\mathrm{u}}(\alpha)+\overline{\mathrm{v}}(\alpha), \\
& (\underline{\mathrm{ku}})(\alpha)=\mathrm{k} \underline{\mathrm{u}}(\alpha),(\overline{\mathrm{ku}})(\alpha)=\mathrm{k} \overline{\mathrm{u}}(\alpha), \text { ifk } \geq 0 \\
& (\underline{\mathrm{ku}})(\alpha)=\mathrm{k} \overline{\mathrm{u}}(\alpha),(\overline{\mathrm{ku}})(\alpha)=\mathrm{ku}(\alpha), \text { ifk }<0
\end{aligned}
$$

The collection of all such fuzzy numbers with addition and multiplication as defined by Equations (1) and (2) is denoted by $E^{1}$ and is a convex cone. Next, we will define the fuzzy function notation and a metric $D$ in $E^{1}$ (Goetschel \& Voxman, 1986).

Definition 2.3 For arbitrary fuzzy numbers $\mathrm{u}=(\underline{\mathrm{u}}(\alpha), \overline{\mathrm{u}}(\alpha)), \mathrm{v}=(\underline{\mathrm{v}}(\alpha), \overline{\mathrm{v}}(\alpha))$, the quantity

$$
\mathrm{D}(\mathrm{u}, \mathrm{v})=\max \left\{\sup _{0 \leq \alpha \leq 1}|\underline{\mathrm{u}}(\alpha)-\underline{\mathrm{v}}(\alpha)|, \sup _{0 \leq \alpha \leq 1}|\overline{\mathrm{u}}(\alpha)-\overline{\mathrm{v}}(\alpha)|\right\}
$$

is the distance between $u$ and $v$. This metric is equivalent to the one used by Puri and Ralescu (1983) and Kaleva (1987). It is shown (Puri \& Ralescu, 1986) that $\left(E^{1}, D\right)$ is a complete metric space.

Definition 2.4 A fuzzy function $\mathrm{f}: \mathrm{R}^{1} \rightarrow \mathrm{E}^{1}$ is said to be continuous if for arbitrary fixed $\mathrm{x}_{0} \in \mathrm{R}^{1}$ and $\varepsilon>0$ there exists $\delta>0$ such that

$$
\text { if }\left|\mathrm{x}-\mathrm{x}_{0}\right|<\delta \text {, then } \mathrm{D}\left(\mathrm{f}(\mathrm{x}), \mathrm{f}\left(\mathrm{x}_{0}\right)\right)<\varepsilon
$$

Throughout this work we also consider fuzzy functions which are defined only over a finite interval $[a, b]$ (we simply replace $\mathrm{R}^{1}$ by $[\mathrm{a}, \mathrm{b}]$ in definition 2.4$)$.

\section{Definition 2.5 (Mehrkanoon, Suleiman, \& Majid, 2009)}

The Seikkala derivative $\mathrm{f}^{\prime}(\mathrm{x})$ of a fuzzy function $\mathrm{f}$ is defined by

$$
\left[\mathrm{f}^{\prime}(\mathrm{x})\right]_{\alpha}=\left[\underline{f}^{\prime}(\mathrm{x} ; \alpha), \overline{\mathrm{f}}^{\prime}(\mathrm{x} ; \alpha)\right], \alpha \in(0,1]
$$

where prime symbol denote the derivative with respect to $x$.

Following the idea of Bede and Gal in 2004 and 2005, Chalco-Cano and Roman-Flores (2008) define the fuzzy lateral $H$-derivative for a fuzzy function $\mathrm{f}: \mathrm{I} \rightarrow \mathrm{E}^{1}$ as follows:

Definition 2.6 (Mehrkanoon, Suleiman, \&Majid, 2009)

Let $\mathrm{f}: \mathrm{I} \rightarrow \mathrm{E}^{1}$ be a fuzzy function and $\mathrm{x}_{0} \in \mathrm{I} \subseteq \mathrm{R}$, then $\mathrm{f}$ is differentiable at $x_{0}$, if

(I) there exist an element $f^{\prime}\left(x_{0}\right) \in E^{1}$, such that for all $h>0$ sufficiently small, there are $f\left(x_{0}+h\right)-f\left(x_{0}\right)$; $\mathrm{f}\left(\mathrm{x}_{0}\right)-\mathrm{f}\left(\mathrm{x}_{0}-\mathrm{h}\right)$ and

$$
\lim _{h \rightarrow 0^{+}} \frac{f\left(x_{0}+h\right)-f\left(x_{0}\right)}{h}=\lim _{h \rightarrow 0^{+}} \frac{f\left(x_{0}\right)-f\left(x_{0}-h\right)}{h}=f^{\prime}\left(x_{0}\right)
$$

or

(II) there exist an element $f^{\prime}\left(x_{0}\right) \in E^{1}$, such that for all $h<0$ sufficiently small, there are $f\left(x_{0}+h\right)-f\left(x_{0}\right)$; $\mathrm{f}\left(\mathrm{x}_{0}\right)-\mathrm{f}\left(\mathrm{x}_{0}-\mathrm{h}\right)$ and

$$
\lim _{h \rightarrow 0^{-}} \frac{f\left(x_{0}+h\right)-f\left(x_{0}\right)}{h}=\lim _{h \rightarrow 0^{-}} \frac{f\left(x_{0}\right)-f\left(x_{0}-h\right)}{h}=f^{\prime}\left(x_{0}\right)
$$

where the relation (I) is the classical definition of the fuzzy H-derivative (or differentiability in the sense of Hukuhara).

\section{HAM for Solving Fuzzy Volterra Integro-Differential Equations (FVIDEs)}

In this section, we shall apply HAM for solving linear and nonlinear fuzzy Volterra integro-differential equations (FVIDEs)

$$
F^{\prime}(x)=f(x)+\lambda \int_{0}^{x} K(x, t) F(t) d t
$$




$$
F^{\prime}(x)=f(x)+\lambda \int_{0}^{x} K(x, t, F(t)) d t
$$

under the initial condition $F_{0}=F(0)=(\underline{F}(0, \alpha), \bar{F}(0, \alpha))=(0,0)$ where $F^{\prime}(x)=\frac{d}{d x} F(x), \quad f:[0, b] \rightarrow E^{1} \quad$ is continuous fuzzy function, $\mathrm{K}$ is arbitrary continuous function over the regions

$$
\Omega=\{(\mathrm{x}, \mathrm{t}) \mid 0 \leq \mathrm{t} \leq \mathrm{x} \leq \mathrm{b}\} \text { and } \Delta=\left\{(\mathrm{x}, \mathrm{t}, \mathrm{F}(\mathrm{x})) \mid 0 \leq \mathrm{t} \leq \mathrm{x} \leq \mathrm{b}, \mathrm{F}(\mathrm{x}) \in \mathrm{E}^{1}\right\}
$$

respectively, and $\mathrm{F}$ is to be determined.

In the following, we shall follow the same method that proposed by Bede (2008), and Chalco-Cano and Roman-Flores (2008) in the fuzzy differential equations to reduce FVIDEs to a crisp systems of integro-differential equations (IDEs) using relations (4) and (5) in definition (2.6).

Let us recall the proposed method. We denote the $\alpha$-level set of $F$, $f$ and $K$ by

$$
\begin{aligned}
{[\mathrm{F}(\mathrm{x})]_{\alpha} } & =[\mathrm{F}(\mathrm{x} ; \alpha), \overline{\mathrm{F}}(\mathrm{x} ; \alpha)], \\
{[\mathrm{f}(\mathrm{x})]_{\alpha} } & =[\underline{\mathrm{f}}(\mathrm{x} ; \alpha), \overline{\mathrm{f}}(\mathrm{x} ; \alpha)],
\end{aligned}
$$

and

$$
\left[\mathrm{K}(\mathrm{x}, \mathrm{t}, \mathrm{F}(\mathrm{t})]_{\alpha}=[\mathrm{K}(\mathrm{x}, \mathrm{t}, \underline{\mathrm{F}}(\mathrm{t}, \alpha)), \mathrm{K}(\mathrm{x}, \mathrm{t}, \overline{\mathrm{F}}(\mathrm{t}, \alpha))]\right.
$$

respectively. Then we have the following two cases:

Case I If we consider $\mathrm{F}^{\prime}(\mathrm{x})$ in the first form (4) of definition (2.6) then we have to solve the following systems of crisp IDEs

$$
\begin{aligned}
& \underline{\mathrm{F}}^{\prime}(\mathrm{x} ; \alpha)=\underline{\mathrm{f}}(\mathrm{x} ; \alpha)+\lambda \int_{0}^{\mathrm{x}} \mathrm{K}(\mathrm{x}, \mathrm{t}) \underline{\mathrm{F}}(\mathrm{t} ; \alpha) \mathrm{dt}, \quad \underline{\mathrm{F}}(0 ; \alpha)=\overline{\mathrm{F}}(0 ; \alpha)=0 \\
& \overline{\mathrm{F}}^{\prime}(\mathrm{x} ; \alpha)=\overline{\mathrm{f}}(\mathrm{x} ; \alpha)+\lambda \int_{0}^{\mathrm{x}} \mathrm{K}(\mathrm{x}, \mathrm{t}) \overline{\mathrm{F}}(\mathrm{t} ; \alpha) \mathrm{dt}
\end{aligned}
$$

for the linear case, and

for the nonlinear case.

$$
\begin{aligned}
& \underline{\mathrm{F}}^{\prime}(\mathrm{x} ; \alpha)=\underline{\mathrm{f}}(\mathrm{x} ; \alpha)+\lambda \int_{0}^{\mathrm{x}} \mathrm{K}(\mathrm{x}, \mathrm{t}, \underline{\mathrm{F}}(\mathrm{t} ; \alpha)) \mathrm{dt}, \underline{\mathrm{F}}(0 ; \alpha)=\overline{\mathrm{F}}(0 ; \alpha)=0 \\
& \overline{\mathrm{F}}^{\prime}(\mathrm{x} ; \alpha)=\overline{\mathrm{f}}(\mathrm{x} ; \alpha)+\lambda \int_{0}^{\mathrm{x}} \mathrm{K}(\mathrm{x}, \mathrm{t}, \overline{\mathrm{F}}(\mathrm{t} ; \alpha)) \mathrm{dt}
\end{aligned}
$$

Case II If we consider $\mathrm{F}^{\prime}(\mathrm{x})$ in the second form (5) of definition (2.6), then we have to solve the following systems of crisp IDEs

$$
\begin{aligned}
& \underline{\mathrm{F}}^{\prime}(\mathrm{x} ; \alpha)=\overline{\mathrm{f}}(\mathrm{x} ; \alpha)+\lambda \int_{0}^{\mathrm{x}} \mathrm{K}(\mathrm{x}, \mathrm{t}) \overline{\mathrm{F}}(\mathrm{t} ; \alpha) \mathrm{dt} \\
& \overline{\mathrm{F}}^{\prime}(\mathrm{x} ; \alpha)=\underline{\mathrm{f}}(\mathrm{x} ; \alpha)+\lambda \int_{0}^{\mathrm{x}} \mathrm{K}(\mathrm{x}, \mathrm{t}) \underline{\mathrm{F}}(\mathrm{t} ; \alpha) \mathrm{dt}(0 ; \alpha)=\overline{\mathrm{F}}(0 ; \alpha)=0
\end{aligned}
$$

for the linear case, and

$$
\begin{aligned}
& \underline{\mathrm{F}}^{\prime}(\mathrm{x} ; \alpha)=\overline{\mathrm{f}}(\mathrm{x} ; \alpha)+\lambda \int_{0}^{\mathrm{x}} \mathrm{K}(\mathrm{x}, \mathrm{t}, \overline{\mathrm{F}}(\mathrm{t} ; \alpha)) \mathrm{dt} \\
& \overline{\mathrm{F}}^{\prime}(\mathrm{x} ; \alpha)=\underline{\mathrm{f}}(\mathrm{x} ; \alpha)+\lambda \int_{0}^{\mathrm{x}} \mathrm{K}(\mathrm{x}, \mathrm{t}, \underline{\mathrm{F}}(\mathrm{t} ; \alpha)) \mathrm{dt}(0 ; \alpha)=\overline{\mathrm{F}}(0 ; \alpha)=0
\end{aligned}
$$

for the nonlinear case.

In our work, we will consider case $I$, hence we have to solve the systems (8) and (9).

For simplicity and without lose of generality of the problem, we assume that the kernels $\mathrm{K}(\mathrm{x}, \mathrm{t}) \geq 0$ on $\Omega$ and $\mathrm{K}(\mathrm{x}, \mathrm{t}, \underline{\varphi}(\mathrm{x})) \geq 0, \mathrm{~K}(\mathrm{x}, \mathrm{t}, \bar{\varphi}(\mathrm{x})) \geq 0$ on $\Delta$.

\subsection{Linear Fuzzy VolterraIntegro-Differential Equations}

For solving system (8) by HAM we first construct the zeroth-order deformation equations: 


$$
\begin{aligned}
(1-\mathrm{p}) \mathrm{L}\left[\underline{\varphi}(\mathrm{x}, \mathrm{p} ; \alpha)-\underline{\mathrm{w}}_{0}(\mathrm{x} ; \alpha)\right] & =\mathrm{p} \hbar \mathrm{H}(\mathrm{x})[\underline{\varphi}(\mathrm{x}, \mathrm{p} ; \alpha)-\underline{\mathrm{f}}(\mathrm{x} ; \alpha) \\
& \left.-\lambda \int_{0}^{\mathrm{x}} \mathrm{K}(\mathrm{x}, \mathrm{t}) \underline{\varphi}(\mathrm{t}, \mathrm{p} ; \alpha) \mathrm{dt}\right] \\
(1-\mathrm{p}) \mathrm{L}\left[\bar{\varphi}(\mathrm{x}, \mathrm{p} ; \alpha)-\overline{\mathrm{w}}_{0}(\mathrm{x} ; \alpha)\right] & =\mathrm{p} \hbar \mathrm{H}(\mathrm{x})\left[\overline{\mathrm{F}}^{\prime}(\mathrm{x}, \mathrm{p} ; \alpha)-\overline{\mathrm{f}}(\mathrm{x} ; \alpha)\right. \\
& \left.-\lambda \int_{0}^{\mathrm{x}} \mathrm{K}(\mathrm{x}, \mathrm{t}) \overline{\mathrm{F}}(\mathrm{t}, \mathrm{p} ; \alpha) \mathrm{dt}\right]
\end{aligned}
$$

where $\mathrm{p} \in[0,1]$ is the embedding parameter, $\hbar$ is nonzero auxiliary parameter, $\mathrm{L}$ is an auxiliary linear operator, $\mathrm{H}(\mathrm{x})$ is an auxiliary function, $\underline{\mathrm{w}}_{0}(\mathrm{x} ; \alpha)$ and $\mathrm{w}_{0}(\mathrm{x} ; \alpha)$ are initial guesses of $\underline{\mathrm{F}}(\mathrm{x} ; \alpha)$ and $\mathrm{F}(\mathrm{x} ; \alpha)$

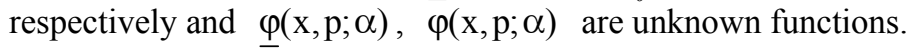

Differentiating the zeroth-order deformation Equations (12) $\mathrm{m}$ times with respect to the embedding parameter $\mathrm{p}$ and dividing them by $\mathrm{m}$ ! and finally setting $\mathrm{p}=0$, we obtain the so called $\mathrm{m}-$ th order deformation equations

$$
\begin{gathered}
\mathrm{L}\left[\underline{\mathrm{u}}_{\mathrm{m}}(\mathrm{x} ; \alpha)-\chi_{\mathrm{m}} \underline{\mathrm{u}}_{\mathrm{m}-1}(\mathrm{x} ; \alpha)\right]=\hbar \mathrm{H}(\mathrm{x})\left[\underline{\mathrm{u}}_{\mathrm{m}-1}^{\prime}(\mathrm{x} ; \alpha)-\left(1-\chi_{\mathrm{m}}\right) \underline{\mathrm{f}}(\mathrm{x} ; \alpha)\right. \\
\left.-\lambda \int_{0}^{\mathrm{x}} \mathrm{K}(\mathrm{x}, \mathrm{t}) \underline{\mathrm{u}}_{\mathrm{m}-1}(\mathrm{t} ; \alpha) \mathrm{dt}\right] \\
\underline{\underline{u}}_{\mathrm{m}}(0 ; \alpha)=0 \\
\mathrm{~L}\left[\overline{\mathrm{u}}_{\mathrm{m}}(\mathrm{x} ; \alpha)-\chi_{\mathrm{m}} \overline{\mathrm{u}}_{\mathrm{m}-1}(\mathrm{x} ; \alpha)\right]=\hbar \mathrm{H}(\mathrm{x})\left[\overline{\mathrm{u}}_{\mathrm{m}-1}^{\prime}(\mathrm{x} ; \alpha)-\left(1-\chi_{\mathrm{m}}\right) \overline{\mathrm{f}}(\mathrm{x} ; \alpha)\right. \\
\left.-\lambda \int_{0}^{\mathrm{x}} \mathrm{K}(\mathrm{x}, \mathrm{t}) \overline{\mathrm{u}}_{\mathrm{m}-1}(\mathrm{t} ; \alpha) \mathrm{dt}\right] \\
\overline{\mathrm{u}}_{\mathrm{m}}(0 ; \alpha)=0
\end{gathered}
$$

Choose the auxiliary linear operator as $\mathrm{L}=\frac{\partial}{\partial \mathrm{x}}$, the initial guesses $\underline{\mathrm{w}}_{0}=\underline{\mathrm{u}}_{0}=0, \overline{\mathrm{w}}_{0}=\overline{\mathrm{u}}_{0}=0$ and the auxiliary function $\mathrm{H}(\mathrm{x})=1$.

By taking $L^{-1}=\int_{0}^{x}$ on both sides of equations (13) and (14) then we obtain the following iteration forms

$$
\begin{aligned}
& \underline{\mathrm{u}}_{1}(\mathrm{x} ; \alpha)=-\hbar \int_{0}^{\mathrm{x}} \underline{\mathrm{f}}(\mathrm{s}, \alpha) \mathrm{ds} \\
& \overline{\mathrm{u}}_{1}(\mathrm{x} ; \alpha)=-\hbar \int_{0}^{\mathrm{x}} \overline{\mathrm{f}}(\mathrm{s}, \alpha) \mathrm{ds}
\end{aligned}
$$

and

$$
\begin{aligned}
& \underline{\mathrm{u}}_{\mathrm{m}}(\mathrm{x} ; \alpha)=(1+\hbar) \underline{\mathrm{u}}_{\mathrm{m}-1}(\mathrm{x} ; \alpha)-\hbar \lambda \int_{0}^{\mathrm{x}} \int_{0}^{\mathrm{s}} \mathrm{K}(\mathrm{s}, \mathrm{t}) \underline{\mathrm{u}}_{\mathrm{m}-1}(\mathrm{t} ; \alpha) \mathrm{dtds} \\
& \overline{\mathrm{u}}_{\mathrm{m}}(\mathrm{x} ; \alpha)=(1+\hbar) \overline{\mathrm{u}}_{\mathrm{m}-1}(\mathrm{x} ; \alpha)-\hbar \lambda \int_{0}^{\mathrm{x}} \int_{0}^{\mathrm{s}} \mathrm{K}(\mathrm{s}, \mathrm{t}) \overline{\mathrm{u}}_{\mathrm{m}-1}(\mathrm{t} ; \alpha) \mathrm{dtds}
\end{aligned}
$$

Hence, the homotopy solution series is given by

$$
\begin{aligned}
& \underline{\mathrm{F}}(\mathrm{x} ; \alpha)=\sum_{\mathrm{m}=1}^{\infty} \underline{\mathrm{u}}_{\mathrm{m}}(\mathrm{x} ; \alpha) \\
& \overline{\mathrm{F}}(\mathrm{x} ; \alpha)=\sum_{\mathrm{m}=1}^{\infty} \overline{\mathrm{u}}_{\mathrm{m}}(\mathrm{x} ; \alpha)
\end{aligned}
$$

therefore the approximation solution series of order $n$ is

$$
\begin{aligned}
& \underline{\mathrm{F}}_{\mathrm{n}}(\mathrm{x} ; \alpha)=\sum_{\mathrm{m}=1}^{\mathrm{n}} \underline{\mathrm{u}}_{\mathrm{m}}(\mathrm{x} ; \alpha) \\
& \overline{\mathrm{F}}_{\mathrm{n}}(\mathrm{x} ; \alpha)=\sum_{\mathrm{m}=1}^{\mathrm{n}} \overline{\mathrm{u}}_{\mathrm{m}}(\mathrm{x} ; \alpha)
\end{aligned}
$$

\subsection{Nonlinear Fuzzy Volterra Integro-Differential Equations}

For solving system (9) by HAM we first construct the zeroth-order deformation equations

where

$$
\begin{aligned}
& (1-\mathrm{p}) \mathrm{L}\left[\underline{\varphi}(\mathrm{x}, \mathrm{p} ; \alpha)-\underline{\mathrm{w}}_{0}(\mathrm{x} ; \alpha)\right]=\mathrm{p} \hbar \mathrm{H}(\mathrm{x}) \mathrm{N}[\underline{\varphi}(\mathrm{x}, \mathrm{p} ; \alpha)] \\
& (1-\mathrm{p}) \mathrm{L}\left[\bar{\varphi}(\mathrm{x}, \mathrm{p} ; \alpha)-\overline{\mathrm{w}}_{0}(\mathrm{x} ; \alpha)\right]=\mathrm{p} \hbar \mathrm{H}(\mathrm{x}) \mathrm{N}[\bar{\varphi}(\mathrm{x}, \mathrm{p} ; \alpha)]
\end{aligned}
$$




$$
\mathrm{N}[\underline{\varphi}(\mathrm{x}, \mathrm{p} ; \alpha)]=\underline{\varphi}(\mathrm{x}, \mathrm{p} ; \alpha)-\underline{\mathrm{f}}(\mathrm{x} ; \alpha)-\lambda \int_{0}^{\mathrm{x}} \mathrm{K}(\mathrm{x}, \mathrm{t}, \underline{\varphi}(\mathrm{t}, \mathrm{p} ; \alpha)) \mathrm{dt}
$$

and

$$
\mathrm{N}[\bar{\varphi}(\mathrm{x}, \mathrm{p} ; \alpha)]=\bar{\varphi}^{\prime}(\mathrm{x}, \mathrm{p} ; \alpha)-\overline{\mathrm{f}}(\mathrm{x} ; \alpha)-\lambda \int_{0}^{\mathrm{x}} \mathrm{K}(\mathrm{x}, \mathrm{t}, \bar{\varphi}(\mathrm{t}, \mathrm{p} ; \alpha)) \mathrm{dt}
$$

The corresponding $\mathrm{m}$-th order deformation equations reads:

$$
\begin{gathered}
\mathrm{L}\left[\underline{\mathrm{u}}_{\mathrm{m}}(\mathrm{x} ; \alpha)-\chi_{\mathrm{m}} \underline{\mathrm{u}}_{\mathrm{m}-1}(\mathrm{x} ; \alpha)\right]=\hbar \mathrm{H}(\mathrm{x}) \mathrm{R}_{\mathrm{m}}\left[\underline{\mathrm{u}}_{\mathrm{m}-1}(\mathrm{x} ; \alpha)\right] \\
\underline{\mathrm{u}}_{\mathrm{m}}(0 ; \alpha)=0
\end{gathered}
$$

and

$$
\begin{gathered}
\mathrm{L}\left[\overline{\mathrm{u}}_{\mathrm{m}}(\mathrm{x} ; \alpha)-\chi_{\mathrm{m}} \overline{\mathrm{u}}_{\mathrm{m}-1}(\mathrm{x} ; \alpha)\right]=\hbar \mathrm{H}(\mathrm{x}) \mathrm{R}_{\mathrm{m}}\left[\overline{\mathrm{u}}_{\mathrm{m}-1}(\mathrm{x} ; \alpha)\right] \\
\overline{\mathrm{u}}_{\mathrm{m}}(0 ; \alpha)=0
\end{gathered}
$$

where

$$
\mathrm{R}_{\mathrm{m}}\left[\underline{\mathrm{u}}_{\mathrm{m}-1}(\mathrm{x} ; \alpha)\right]=\underline{\mathrm{u}}_{\mathrm{m}-1}^{\prime}(\mathrm{x} ; \alpha)-\left(1-\chi_{\mathrm{m}}\right) \underline{\mathrm{f}}(\mathrm{x} ; \alpha)-\lambda \int_{0}^{\mathrm{x}} \underline{\mathrm{K}}_{\mathrm{m}-1}(\mathrm{x}, \mathrm{t} ; \alpha) \mathrm{dt}
$$

and

where

$$
\mathrm{R}_{\mathrm{m}}\left[\overline{\mathrm{u}}_{\mathrm{m}-1}(\mathrm{x} ; \alpha)\right]=\overline{\mathrm{u}}_{\mathrm{m}-1}(\mathrm{x} ; \alpha)-\left(1-\chi_{\mathrm{m}}\right) \overline{\mathrm{f}}(\mathrm{x} ; \alpha)-\lambda \int_{0}^{\mathrm{x}} \overline{\mathrm{K}}_{\mathrm{m}-1}(\mathrm{x}, \mathrm{t} ; \alpha) \mathrm{dt}
$$

$$
\begin{aligned}
& \underline{\mathrm{K}}_{\mathrm{m}-1}(\mathrm{x}, \mathrm{t} ; \alpha)=\left.\frac{1}{(\mathrm{~m}-1) !} \frac{\partial^{\mathrm{m}-1}}{\partial \mathrm{p}^{\mathrm{m}-1}} \mathrm{~K}(\mathrm{x}, \mathrm{t}, \underline{\varphi}(\mathrm{t}, \mathrm{p} ; \alpha))\right|_{\mathrm{p}=0} \\
& \overline{\mathrm{K}}_{\mathrm{m}-1}(\mathrm{x}, \mathrm{t} ; \alpha)=\left.\frac{1}{(\mathrm{~m}-1) !} \frac{\partial^{\mathrm{m}-1}}{\partial \mathrm{p}^{\mathrm{m}-1}} \mathrm{~K}(\mathrm{x}, \mathrm{t}, \bar{\varphi}(\mathrm{t}, \mathrm{p} ; \alpha))\right|_{\mathrm{p}=0}
\end{aligned}
$$

Choose the auxiliary linear operator as $L=\frac{\partial}{\partial x}$ and the auxiliary function $H(x)=1$.

By taking $L^{-1}=\int_{0}^{x}$ on both sides of equations (22) and (23) we obtain the following iteration forms

and

$$
\begin{aligned}
& \underline{\mathrm{u}}_{1}(\mathrm{x} ; \alpha)=\hbar \underline{\mathrm{u}}_{0}(\mathrm{x} ; \alpha)-\hbar \int_{0}^{\mathrm{x}} \underline{\mathrm{f}}(\mathrm{s}, \alpha) \mathrm{ds}-\lambda \hbar \int_{0}^{\mathrm{x}} \int_{0}^{\mathrm{s}} \underline{\mathrm{K}}_{0}(\mathrm{~s}, \mathrm{t} ; \alpha) \mathrm{dtds} \\
& \overline{\mathrm{u}}_{1}(\mathrm{x} ; \alpha)=\hbar \overline{\mathrm{u}}_{0}(\mathrm{x} ; \alpha)-\hbar \int_{0}^{\mathrm{x}} \overline{\mathrm{f}}(\mathrm{s}, \alpha) \mathrm{ds}-\lambda \hbar \int_{0}^{\mathrm{x}} \int_{0}^{\mathrm{s}} \overline{\mathrm{K}}_{0}(\mathrm{~s}, \mathrm{t} ; \alpha) \mathrm{dtds}
\end{aligned}
$$

where

$$
\begin{aligned}
& \underline{\mathrm{u}}_{\mathrm{m}}(\mathrm{x} ; \alpha)=(1+\hbar) \underline{\mathrm{u}}_{\mathrm{m}-1}(\mathrm{x} ; \alpha)-\hbar \lambda \int_{0}^{\mathrm{x}} \int_{0}^{\mathrm{s}} \underline{\mathrm{K}}_{\mathrm{m}-1}(\mathrm{~s}, \mathrm{t} ; \alpha) \mathrm{dtds} \\
& \overline{\mathrm{u}}_{\mathrm{m}}(\mathrm{x} ; \alpha)=(1+\hbar) \overline{\mathrm{u}}_{\mathrm{m}-1}(\mathrm{x} ; \alpha)-\hbar \lambda \int_{0}^{\mathrm{x}} \int_{0}^{\mathrm{s}} \overline{\mathrm{K}}_{\mathrm{m}-1}(\mathrm{~s}, \mathrm{t} ; \alpha) \mathrm{dtds}, \mathrm{m} \geq 2
\end{aligned}
$$

and

$$
\underline{\mathrm{K}}_{0}(\mathrm{~s}, \mathrm{t} ; \alpha)=\mathrm{K}\left(\mathrm{s}, \mathrm{t}, \underline{\mathrm{u}}_{0}(\mathrm{t} ; \alpha)\right)
$$

$$
\overline{\mathrm{K}}_{0}(\mathrm{~s}, \mathrm{t} ; \alpha)=\mathrm{K}\left(\mathrm{s}, \mathrm{t}, \overline{\mathrm{u}}_{0}(\mathrm{t} ; \alpha)\right)
$$

Hence, the homotopy solution series is given by

$$
\begin{aligned}
& \underline{\mathrm{F}}(\mathrm{x} ; \alpha)=\underline{\mathrm{u}}_{0}(\mathrm{x} ; \alpha)+\sum_{\mathrm{m}=1}^{\infty} \underline{\mathrm{u}}_{\mathrm{m}}(\mathrm{x} ; \alpha) \\
& \overline{\mathrm{F}}(\mathrm{x} ; \alpha)=\overline{\mathrm{u}}_{0}(\mathrm{x} ; \alpha)+\sum_{\mathrm{m}=1}^{\infty} \overline{\mathrm{u}}_{\mathrm{m}}(\mathrm{x} ; \alpha)
\end{aligned}
$$

therefore the approximation solution series of order $\mathrm{n}$ is

\section{Numerical Examples}

$$
\begin{aligned}
& \underline{\mathrm{F}}_{\mathrm{n}}(\mathrm{x} ; \alpha)=\underline{\mathrm{u}}_{0}(\mathrm{x} ; \alpha)+\sum_{\mathrm{m}=1}^{\mathrm{n}} \underline{\mathrm{u}}_{\mathrm{m}}(\mathrm{x} ; \alpha) \\
& \overline{\mathrm{F}}_{\mathrm{n}}(\mathrm{x} ; \alpha)=\overline{\mathrm{u}}_{0}(\mathrm{x} ; \alpha)+\sum_{\mathrm{m}=1}^{\mathrm{n}} \overline{\mathrm{u}}_{\mathrm{m}}(\mathrm{x} ; \alpha)
\end{aligned}
$$

In this section, we examine our method by giving two numerical examples with known exact solutions. 


\section{Example 4.1}

Consider the linear FVIDE with

$$
\mathrm{K}(\mathrm{x}, \mathrm{t})=\mathrm{xt}, \mathrm{b}=1, \lambda=1
$$

and

$$
\begin{gathered}
\mathrm{f}(\mathrm{x} ; \alpha)=\alpha\left(1-\frac{1}{3} \mathrm{x}^{4}\right) \\
\overline{\mathrm{f}}(\mathrm{x} ; \alpha)=2-\alpha-\frac{1}{3} \mathrm{x}^{4}(2-\alpha)
\end{gathered}
$$

The exact solution in this case is given by

From Equations (15) and (16) we obtain

$$
\begin{aligned}
& \underline{F}(x ; \alpha)=\alpha x \\
& \bar{F}(x ; \alpha)=(2-\alpha) x
\end{aligned}
$$

$$
\begin{aligned}
& \underline{\mathrm{u}}_{1}(\mathrm{x} ; \alpha)=-\hbar \alpha\left(\mathrm{x}-\frac{1}{15} \mathrm{x}^{5}\right), \\
& \underline{\mathrm{u}}_{2}(\mathrm{x} ; \alpha)=-(1+\hbar) \hbar \alpha\left(\mathrm{x}-\frac{1}{15} \mathrm{x}^{5}\right)-\hbar^{2} \alpha\left(\frac{1}{945} \mathrm{x}^{9}-\frac{1}{15} \mathrm{x}^{5}\right), \\
& \underline{\mathrm{u}}_{3}(\mathrm{x} ; \alpha)=(1+\hbar)\left\{(-1-\hbar) \hbar \alpha\left(\mathrm{x}-\frac{1}{15} \mathrm{x}^{5}\right)-\hbar^{2} \alpha\left(\frac{1}{945} \mathrm{x}^{9}-\frac{1}{15} \mathrm{x}^{5}\right)\right\}-\hbar\left(-\frac{8736386729695573}{1180591620717411303424} \hbar^{2} \alpha \mathrm{x}^{13},\right. \\
& \left.+\left(\frac{1}{9}\left(\frac{1}{105}+\frac{1}{105} \hbar\right) \hbar \alpha+\frac{1}{945} \hbar^{2} \alpha\right) \mathrm{x}^{9}+\left(-\frac{1}{15}-\frac{1}{15} \hbar\right) \hbar \alpha \mathrm{x}^{5}\right) \\
& \underline{\mathrm{u}}_{4}(\mathrm{x} ; \alpha)=\left(-\hbar \alpha-3 \hbar^{2} \alpha-3 \hbar^{3} \alpha-\hbar^{4} \alpha\right) \mathrm{x}+\left(\frac{1}{15} \hbar \alpha+\frac{2}{5} \hbar^{2} \alpha+\frac{4}{15} \hbar^{4} \alpha+\frac{3}{5} \hbar^{3} \alpha\right) \mathrm{x}^{5}\left(-\frac{2}{315} \hbar^{4} \alpha\right. \\
& \left.-\frac{1}{105} \hbar^{3} \alpha-\frac{1}{315} \hbar^{2} \alpha\right) \mathrm{x}^{9}+\left(\frac{3541774862152233864203}{159539248665647376488202240} \hbar^{3} \alpha+\frac{1574122160956548389209}{531797495552515792162734080} \hbar^{4} \alpha\right) \mathrm{x}^{13}, \\
& -\frac{43853235349060013}{151115727451828646838272} \hbar^{4} \alpha \mathrm{x}^{17} \\
& \underline{\mathrm{u}}_{5}(\mathrm{x} ; \alpha)=\left(-\hbar \alpha-6 \hbar^{3} \alpha-4 \hbar^{4} \alpha-4 \hbar^{2} \alpha-\hbar^{5} \alpha\right) \mathrm{x}+\left(\frac{1}{15} \hbar \alpha+\frac{8}{15} \hbar^{2} \alpha+\frac{6}{5} \hbar^{3} \alpha+\frac{1}{3} \hbar^{5} \alpha+\frac{16}{15} \hbar^{4} \alpha\right) \mathrm{x}^{5} \\
& -\left(\frac{4}{945} \hbar^{2} \alpha-\frac{8}{315} \hbar^{4} \alpha-\frac{2}{105} \hbar^{3} \alpha-\frac{2}{189} \hbar^{5} \alpha\right) \mathrm{x}^{9}+\left(\frac{3541774862152233864203}{7976962433282368824101120} \hbar^{3} \alpha\right. \\
& \left.+\frac{1574122160956548389209}{13294937388803948040683520} \hbar^{4} \alpha+\frac{5902958103587056471051}{79769624332823688244101120} \hbar^{5} \alpha\right) x^{13}-\left(\frac{604462909807314567905837}{5207361076446730368574921113600} \hbar^{4} \alpha\right. \text {, and } \\
& \left.-\frac{755578637259143214744109}{5207361076467730385574921113600} \hbar^{5} \alpha\right) \mathrm{x}^{17}+\frac{2813641165252981}{38685626227668133590597632} \hbar^{5} \alpha \mathrm{x}^{21} \\
& \overline{\mathrm{u}}_{1}(\mathrm{x} ; \alpha)=-\hbar\left\{\left(-\frac{2}{15}+\frac{1}{15} \alpha\right) \mathrm{x}^{5}+2 \mathrm{x}-\alpha \mathrm{x}\right\}, \\
& \overline{\mathrm{u}}_{2}(\mathrm{x} ; \alpha)=-(1+\hbar) \hbar\left\{\left(-\frac{2}{15}+\frac{1}{15} \alpha\right) \mathrm{x}^{5}+2 \mathrm{x}-\alpha \mathrm{x}\right\}-\hbar\left\{-\frac{1}{63} \hbar\left(-\frac{2}{15}+\frac{1}{15} \alpha\right) \mathrm{x}^{9}-\frac{1}{15} \hbar(2-\alpha) \mathrm{x}^{5}\right\}, \\
& \overline{\mathrm{u}}_{3}(\mathrm{x} ; \alpha)=\left(\hbar \alpha-2 \hbar^{3}-2 \hbar+\hbar^{3} \alpha+2 \hbar^{2} \alpha-4 \hbar^{2}\right) \mathrm{x}+\frac{1}{15}\left(2 \hbar \mathrm{x}^{5}+6 \hbar^{3} \mathrm{x}^{5}-3 \hbar^{3} \alpha \mathrm{x}^{5}-\hbar \alpha \mathrm{x}^{5}-4 \hbar^{2} \alpha \mathrm{x}^{5}\right. \\
& \left.+8 \hbar^{2} \mathrm{x}^{5}\right)-\left(\frac{4}{9} 45 \hbar^{2}+\frac{1}{315} \hbar^{3} \alpha+\frac{2}{945} \hbar^{2} \alpha-\frac{2}{315} \hbar^{3}\right) \mathrm{x}^{9}+\frac{1}{135135}\left(2 \hbar^{3}-\hbar^{3} \alpha\right) \mathrm{x}^{13} \\
& \overline{\mathrm{u}}_{4}(\mathrm{x} ; \alpha)=\left(\hbar \alpha-2 \hbar-2 \hbar^{4}+3 \hbar^{2} \alpha+\hbar^{4} \alpha+3 \hbar^{3} \alpha-6 \hbar^{2}-6 \hbar^{3}\right) \mathrm{x}+\left(\frac{2}{15} \hbar-\frac{4}{15} \hbar^{4} \alpha+8 / 15 \hbar^{4}-\frac{3}{5} \hbar^{3} \alpha\right. \\
& \left.-\frac{1}{15} \hbar \alpha-\frac{2}{5} \hbar^{2} \alpha+\frac{4}{5} \hbar^{2}+\frac{6}{5} \hbar^{3}\right) \mathrm{x}^{5}-\left(\frac{2}{105} \hbar^{3}+\frac{1}{315} \hbar^{2} \alpha-\frac{4}{315} \hbar^{4}+\frac{2}{315} \hbar^{4} \alpha+\frac{1}{105} \hbar^{3}-\frac{2}{315} \hbar^{2}\right) \mathrm{x}^{9} \\
& +\left(\frac{2}{45045} \hbar^{3}-\frac{1}{45045} \hbar^{3} \alpha-\frac{4}{135135} \hbar^{4} \alpha+\frac{8}{135135} \hbar^{4}\right) \mathrm{x}^{13}+\frac{1644496325589755}{56668397794435572564352} \hbar^{4} \alpha \mathrm{x}^{17}-\frac{1644496325589755}{28334198897217871282176} \hbar^{4} \mathrm{x}^{17} \\
& \overline{\mathrm{u}}_{5}(\mathrm{x} ; \alpha)=\left(\hbar \alpha-8 \hbar^{4}-2 \hbar^{5}-2 \hbar-8 \hbar^{2}+\hbar^{5} \alpha+4 \hbar^{4} \alpha-12 \hbar^{3}+6 \hbar^{3} \alpha \mathrm{x}+4 \hbar^{2} \alpha\right) \mathrm{x} \\
& +\left(\frac{2}{15} \hbar+\frac{2}{3} \hbar^{5}-\frac{1}{3} \hbar^{5} \alpha+\frac{32}{15} \hbar^{4}-\frac{16}{15} \hbar^{4} \alpha+\frac{12}{5} \hbar^{3}+\frac{16}{15} \hbar^{2}-\frac{1}{15} \hbar \alpha-\frac{6}{5} \hbar^{3} \alpha-\frac{8}{15} \hbar^{2} \alpha\right) \mathrm{x}^{5} \\
& -\left(\frac{8}{945} \hbar^{2}-\frac{16}{315} \hbar^{4}+\frac{2}{189} \hbar^{5} \alpha-\frac{4}{189} \hbar^{5}-\frac{4}{105} \hbar^{3}+\frac{4}{945} \hbar^{2} \alpha+\frac{2}{105} \hbar^{3} \alpha+\frac{8}{315} \hbar^{4} \alpha\right) \mathrm{x}^{9} \\
& +\left(\frac{4}{45045} \hbar^{3}-\frac{2}{45045} \hbar^{3} \alpha-\frac{16}{135135} \hbar^{4} \alpha+\frac{32}{135135} \hbar^{4}-\frac{2}{27027} \hbar^{5} \alpha+4 / 27027 \hbar^{5}\right) x^{13} \\
& +\left(\frac{75557863725914322890873}{32546066277920648035932569600} \hbar^{4}-\frac{75557863725914322890873}{325460067277920648035932566600} \hbar^{4}+\frac{31482443219130967915219}{216973378185280432023955046400} \hbar^{5} \alpha\right. \\
& \left.-\frac{31482443219130967915219}{10848668092640216011977523200} \hbar^{5}\right) \mathrm{x}^{17}+\left(\frac{1758525728283113}{12089258196146291747061760} \hbar^{5}-\frac{17585257282883113}{24178516392292583494123520} \hbar^{5} \alpha\right) \mathrm{x}^{21}
\end{aligned}
$$

Then we approximate $\underline{F}(\mathrm{x} ; \alpha)$ with $\mathrm{n}=5$ by 


$$
\begin{aligned}
& \underline{\mathrm{F}}_{5}(\mathrm{x} ; \alpha)=\left(-5 \hbar \alpha-10 \hbar^{2} \alpha-\hbar^{5} \alpha-5 \hbar^{4} \alpha-10 \hbar^{3} \alpha\right) \mathrm{x}+\left(\frac{1}{3} \hbar \alpha+\frac{1}{3} \hbar^{5} \alpha+\frac{4}{3} \hbar^{2} \alpha+\frac{4}{3} \hbar^{4} \alpha+2 \hbar^{3} \alpha\right) \mathrm{x}^{5} \\
& +\left(-\frac{2}{189} \hbar^{2} \alpha-\frac{2}{189} \hbar^{5} \alpha-\frac{2}{63} \hbar^{3} \alpha-\frac{2}{63} \hbar^{4} \alpha\right) \mathrm{x}^{9}+\left(\frac{5902958103587056471051}{79769624332823688244101120} \hbar^{5} \alpha\right. \\
& \left.+\frac{1686559458167730413719}{11395660618974812606300160} \hbar^{4} \alpha+\frac{2361183241434822560779}{31907849733129475297640448} \hbar^{3} \alpha\right) x^{13} \\
& -\left(\frac{7555786372559143198847287}{5207361076446730368574921113600} \hbar^{4} \alpha+\frac{377789318629571604331517}{2603680538223365184287460556800} \hbar^{5} \alpha\right) x^{17}+\frac{2813641165252981}{386856262227668133590597632} \hbar^{5} \alpha x^{21}
\end{aligned}
$$

with $\mathrm{n}=5$ by

$$
\begin{aligned}
& \overline{\mathrm{F}}_{5}(\mathrm{x} ; \alpha)=\left(\hbar^{5} \alpha+10 \hbar^{3} \alpha+10 \hbar^{2} \alpha-2 \hbar^{5}-10 \hbar-20 \hbar^{3}+5 \hbar^{4} \alpha+5 \hbar \alpha-10 \hbar^{4}-20 \hbar^{2}\right) \mathrm{x} \\
& +\left(\frac{2}{3} \hbar^{5}-\frac{4}{3} \hbar^{2} \alpha-\frac{4}{3} \hbar^{4} \alpha+\frac{8}{3} \hbar^{4}-\frac{1}{3} \hbar \alpha+4 \hbar^{3}-\frac{1}{3} \hbar^{5} \alpha+\frac{8}{3} \hbar^{2}+\frac{2}{3} \hbar-2 \hbar^{3} \alpha\right) \mathrm{x}^{5}-\left(\frac{4}{189} \hbar^{5}\right. \\
& \left.+\frac{2}{63} \hbar^{3} \alpha+\frac{2}{189} \hbar^{5} \alpha+\frac{2}{63} \hbar^{4}-\frac{4}{63} \hbar^{4}+\frac{2}{189} \hbar^{2} \alpha-\frac{4}{189} \hbar^{2}-\frac{4}{63} \hbar^{3}\right) \mathrm{x}^{9}+\left(\frac{4}{27027} \hbar^{5}+\frac{4}{27027} \hbar^{3}\right. \\
& \left.+\frac{8}{27027} \hbar^{4}-\frac{2}{27027} \hbar^{3} \alpha-\frac{4}{27027} \hbar^{4} \alpha-\frac{2}{27027} \hbar^{5} \alpha\right) \mathrm{x}^{13}+\left(\frac{944473296573929049900443}{650920134555841296071865139200} \hbar^{4} \alpha\right. \\
& \left.-\frac{15741221609565484115267}{54243344546320108005988761600} \hbar^{5}-\frac{94447329657392904900443}{325460067277920648035932566600} \hbar^{4}+\frac{15741221609565484115267}{108486689092640216011977523200} \hbar^{5} \alpha\right) \mathrm{x}^{17} \\
& +\left(\frac{1758525728283113}{12089258196146291747061760} \hbar^{5}-\frac{17585257282838113}{24178516392292583494123520} \hbar^{5} \alpha\right) \mathrm{x}^{21}
\end{aligned}
$$

We determine the valid region of $\hbar$ such that the homotopy solution series converges, we plot the $\hbar$-curve of approximate solution $\underline{\mathrm{F}}_{5}(0.5,0.5)$ and $\overline{\mathrm{F}}_{5}(0.5,0.5)$ which is obtained by HAM as shown in Figure 1. From this figure we can find that the valid region of $\hbar$ is the interval $[-1.5,-0.3]$. The error between exact and approximate solution of order 5 obtained by HAM is shown in Table 1.

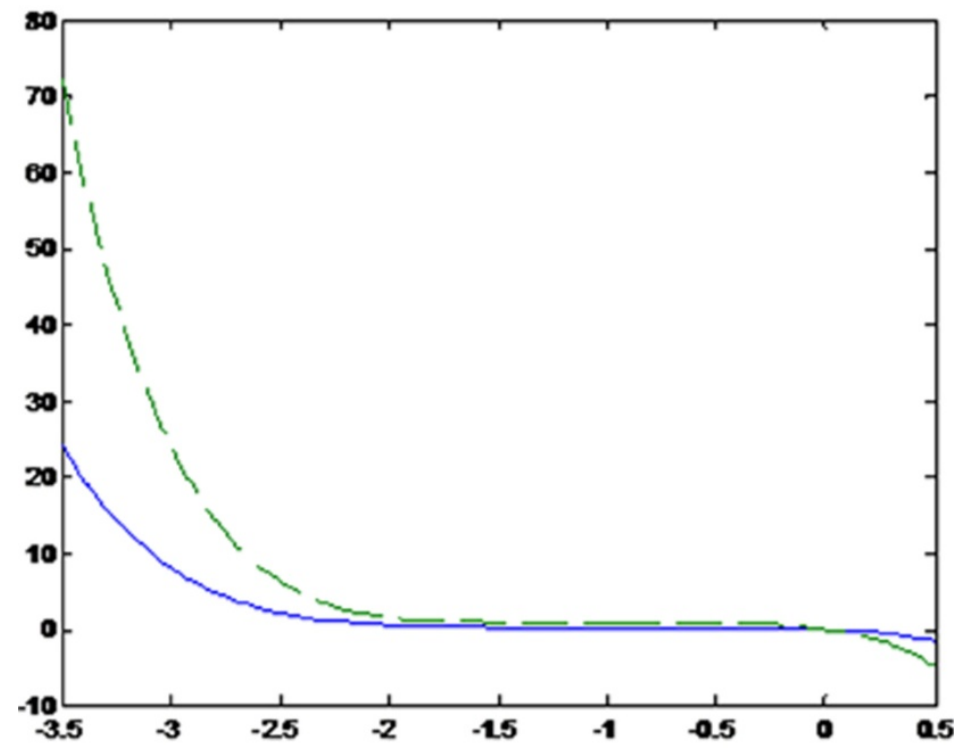

Figure 1. Plot of the $\hbar$-curve of $\underline{\mathrm{F}}_{5}(0.5,0.5)$ and $\dot{\overline{\mathrm{F}}}_{5}\left(0.5, \underline{0.5)}\right.$ dashed dotted line represents $\underline{\mathrm{F}}_{5}(0.5 ; 0.5)$ and solid line represents $\overline{\mathrm{F}}_{5}(0.5 ; 0.5)$

Table 1. The error between exact and approximate solution for different values of $\hbar$

\begin{tabular}{ccccccc}
\hline$x$ & $\hbar=-1.4$ & $\hbar=-1.2$ & $\hbar=-1$ & $\hbar=-0.8$ & $\hbar=-0.6$ & $\hbar=-0.4$ \\
\hline 0 & 0 & 0 & 0 & 0 & 0 & 0 \\
0.1 & $2 . \mathrm{e}-3$ & $6.3987 \mathrm{e}-5$ & $1.4546 \mathrm{e}-31$ & $6.4009 \mathrm{e}-5$ & $2 . \mathrm{e}-3$ & $1.56 \mathrm{e}-2$ \\
0.2 & $4.1 \mathrm{e}-3$ & $1.2759 \mathrm{e}-4$ & $3.0506 \mathrm{e}-25$ & $1.2827 \mathrm{e}-4$ & $4.1 \mathrm{e}-3$ & $3.11 \mathrm{e}-2$ \\
0.3 & $6.1 \mathrm{e}-3$ & $1.8889 \mathrm{e}-4$ & $1.5216 \mathrm{e}-21$ & $1.9408 \mathrm{e}-4$ & $6.2 \mathrm{e}-3$ & $4.67 \mathrm{e}-2$ \\
0.4 & $7.9 \mathrm{e}-3$ & $2.4296 \mathrm{e}-4$ & $6.3975 \mathrm{e}-19$ & $2.6477 \mathrm{e}-4$ & $8.3 \mathrm{e}-3$ & $6.26 \mathrm{e}-2$ \\
0.5 & $9.5 \mathrm{e}-3$ & $2.8047 \mathrm{e}-4$ & $6.9362 \mathrm{e}-17$ & $3.4688 \mathrm{e}-4$ & $1.06 \mathrm{e}-2$ & $7.88 \mathrm{e}-2$ \\
0.6 & $1.05 \mathrm{e}-2$ & $2.8691 \mathrm{e}-4$ & $3.1910 \mathrm{e}-15$ & $4.5145 \mathrm{e}-4$ & $1.31 \mathrm{e}-2$ & $9.60 \mathrm{e}-2$ \\
0.7 & $1.04 \mathrm{e}-2$ & $2.4261 \mathrm{e}-4$ & $8.1247 \mathrm{e}-14$ & $5.9582 \mathrm{e}-4$ & $1.61 \mathrm{e}-2$ & 0.1147
\end{tabular}




\begin{tabular}{ccccccc}
0.8 & $8.9 \mathrm{e}-3$ & $1.2473 \mathrm{e}-4$ & $1.3416 \mathrm{e}-12$ & $8.0633 \mathrm{e}-4$ & $1.98 \mathrm{e}-2$ & 0.1358 \\
0.9 & $5.3 \mathrm{e}-3$ & $8.7951 \mathrm{e}-5$ & $1.5916 \mathrm{e}-11$ & $1.1 \mathrm{e}-3$ & $2.47 \mathrm{e}-2$ & 0.1607 \\
1 & $8.2305 \mathrm{e}-4$ & $4.0630 \mathrm{e}-4$ & $1.4546 \mathrm{e}-10$ & $1.6 \mathrm{e}-3$ & $3.12 \mathrm{e}-2$ & 0.1908 \\
\hline
\end{tabular}

Example 4.2 Consider the nonlinear FVIDE with

$$
\begin{aligned}
& \mathrm{K}(\mathrm{x}, \mathrm{t}, \underline{\mathrm{F}}(\mathrm{t} ; \alpha))=\underline{\mathrm{F}}^{2}(\mathrm{t} ; \alpha) \\
& \mathrm{K}(\mathrm{x}, \mathrm{t}, \overline{\mathrm{F}}(\mathrm{t} ; \alpha))=\overline{\mathrm{F}}^{2}(\mathrm{t} ; \alpha)
\end{aligned}, 0 \leq \mathrm{x} \leq 1 \quad \mathrm{~b}=1, \lambda=1
$$

and

$$
\begin{gathered}
\underline{\mathrm{f}}(\mathrm{x} ; \alpha)=-\frac{1}{5}\left(\alpha^{4}+2 \alpha^{3}+\alpha^{2}\right) \mathrm{x}^{5}+2\left(\alpha^{2}+\alpha\right) \mathrm{x} \\
\overline{\mathrm{f}}(\mathrm{x} ; \alpha)=-\frac{1}{5}\left(16-8 \alpha^{3}-8 \alpha+\alpha^{6}+2 \alpha^{4}+\alpha^{2}\right) \mathrm{x}^{5}+2\left(4-\alpha^{3}-\alpha\right) \mathrm{x}
\end{gathered}
$$

The exact solution in this case is given by

$$
\begin{aligned}
& \underline{\mathrm{F}}(\mathrm{x} ; \alpha)=\left(\alpha^{2}+\alpha\right) \mathrm{x}^{2} \\
& \overline{\mathrm{F}}(\mathrm{x} ; \alpha)=\left(4-\alpha^{3}-\alpha\right) \mathrm{x}^{2}
\end{aligned}
$$

Choose $\underline{\mathrm{u}}_{0}(\mathrm{x} ; \alpha)=\overline{\mathrm{u}}_{0}(\mathrm{x} ; \alpha)=0$ and from equation (28), we obtain

$$
\begin{aligned}
& \underline{\mathrm{u}}_{\mathrm{m}}(\mathrm{x} ; \alpha)=(1+\hbar) \underline{\mathrm{u}}_{\mathrm{m}-1}(\mathrm{x} ; \alpha)-\hbar \lambda \int_{0}^{\mathrm{x}} \int_{0}^{\mathrm{s}} \sum_{\mathrm{i}=0}^{\mathrm{m}-1} \underline{\mathrm{u}}_{\mathrm{i}}(\mathrm{t} ; \alpha) \underline{\mathrm{u}}_{\mathrm{m}-1-\mathrm{i}}(\mathrm{t} ; \alpha) \mathrm{dtds} \\
& \overline{\mathrm{u}}_{\mathrm{m}}(\mathrm{x} ; \alpha)=(1+\hbar) \overline{\mathrm{u}}_{\mathrm{m}-1}(\mathrm{x} ; \alpha)-\hbar \lambda \int_{0}^{\mathrm{x}} \int_{0}^{\mathrm{s}} \sum_{\mathrm{i}=0}^{\mathrm{m}-1} \overline{\mathrm{u}}_{\mathrm{i}}(\mathrm{t} ; \alpha) \overline{\mathrm{u}}_{\mathrm{m}-1-\mathrm{i}}(\mathrm{t} ; \alpha) \mathrm{dtds}
\end{aligned}
$$

From this and (27), we can obtain the first three terms of approximate homotopy solution series as

$$
\begin{gathered}
\underline{\mathrm{u}}_{1}(\mathrm{x} ; \alpha)=\frac{1}{30} \hbar \alpha\left(2 \alpha^{2}+\alpha+\alpha^{3}\right) \mathrm{x}^{6}-\hbar \alpha(1+\alpha) \mathrm{x}^{2}, \\
\underline{\mathrm{u}}_{2}(\mathrm{x} ; \alpha)=\frac{1}{30}(1+\hbar) \hbar \alpha\left(2 \alpha^{2}+\alpha+\alpha^{3}\right) \mathrm{x}^{6}-(1+\hbar) \hbar \alpha(1+\alpha) \mathrm{x}^{2}, \\
\underline{\mathrm{u}}_{3}(\mathrm{x} ; \alpha)=-\left(\frac{56308742593741}{922337203685475808} \hbar^{3} \alpha^{4}+\frac{56308742593741}{922337203685475808} \hbar^{3} \alpha^{8}+\frac{1}{40950} \hbar^{3} \alpha^{7}+\frac{1}{27300} \hbar^{3} \alpha^{6}+\frac{1}{40950} \hbar^{3} \alpha^{5}\right) \mathrm{x}^{14} \\
+\left(\frac{1}{1350} \hbar^{3} \alpha^{6}+\frac{1}{450} \hbar^{3} \alpha^{5}+\frac{1}{1350} \hbar^{3} \alpha^{3}+\frac{1}{450} \hbar^{3} \alpha^{4}\right) \mathrm{x}^{10}+\left(\frac{1}{15} \hbar \alpha^{3}+\frac{1}{15} \hbar^{2} \alpha^{2}+\frac{2}{15} \hbar^{2} \alpha^{3}+\frac{1}{15} \hbar^{2} \alpha^{4},\right. \\
\left.+\frac{1}{30} \hbar \alpha^{4}+\frac{1}{30} \hbar \alpha^{2}\right) \mathrm{x}^{6}-\left(\hbar \alpha^{2}+\hbar^{3} \alpha+\hbar \alpha+\hbar^{3} \alpha^{2}+2 \hbar^{2} \alpha^{2}+2 \hbar^{2} \alpha\right) \mathrm{x}^{2}
\end{gathered}
$$

and

$$
\begin{gathered}
\overline{\mathrm{u}}_{1}(\mathrm{x} ; \alpha)=\frac{1}{30} \hbar\left(-8 \alpha^{3}+16+\alpha^{2}-8 \alpha+\alpha^{6}+2 \alpha^{4}\right) \mathrm{x}^{6}+\frac{1}{30} \hbar\left(30 \alpha-120+30 \alpha^{3}\right) \mathrm{x}^{2}, \\
\overline{\mathrm{u}}_{2}(\mathrm{x} ; \alpha)=\frac{1}{30}(1+\hbar) \hbar\left(-8 \alpha^{3}+16+2 \alpha^{2}-8 \alpha+\alpha^{6}+2 \alpha^{4}\right) \mathrm{x}^{6}+(1+\hbar) \hbar\left(\alpha-40+\alpha^{3}\right) \mathrm{x}^{2}, \\
\overline{\mathrm{u}}_{3}(\mathrm{x} ; \alpha)=\left(-\frac{32}{20475} \hbar^{3}-\frac{56308742593741}{922337203685475808} \hbar^{3} \alpha^{12}-\frac{5433793660296007}{4611686018427387904} \hbar^{3} \alpha^{4}+\frac{32}{20475} \hbar^{3} \alpha-\frac{4}{6825} \hbar^{3} \alpha^{2}\right. \\
\left.+\frac{2}{6825} \hbar^{3} \alpha^{7}-\frac{1}{1638} \hbar^{3} \alpha^{6}+\frac{2}{6825} \hbar^{3} \alpha^{5}-\frac{1}{27300} \alpha^{3}+\frac{2}{20475} \hbar^{3} \alpha^{9}-\frac{1}{40950} \hbar^{3} \alpha^{10}+\frac{34}{20475} \hbar^{3} \alpha^{3}\right) \mathrm{x}^{14} \\
+\left(\frac{32}{675} \hbar^{3}-\frac{1}{1350} \hbar^{3} \alpha^{9}+\frac{2}{225} \hbar^{3} \alpha^{6}-\frac{49}{1350} \hbar^{3} \alpha^{3}-\frac{8}{225} \hbar^{3} \alpha+\frac{4}{225} \hbar^{3} \alpha^{4}-\frac{1}{450} \hbar^{3} \alpha^{5}-\frac{1}{450} \hbar^{3} \alpha^{7}\right. \\
\left.+\frac{2}{225} \hbar^{3} \alpha^{2}\right) \mathrm{x}^{10}+\left(-\frac{8}{15} \hbar^{2} \alpha+\frac{1}{15} \hbar^{2} \alpha^{2}+\frac{1}{15} \hbar^{2} \alpha^{6}+\frac{1}{30} \hbar \alpha^{6}+\frac{16}{15} \hbar^{2}+\frac{8}{15} \hbar+\frac{2}{15} \hbar^{2} \alpha^{4}-\frac{8}{15} \hbar^{2} \alpha^{3}\right. \\
\left.-\frac{4}{15} \hbar \alpha^{3}+\frac{1}{15} \hbar \alpha^{4}-\frac{4}{15} \hbar \alpha+\frac{1}{30} \hbar \alpha^{2}\right) \mathrm{x}^{6}+\left(2 \hbar^{2} \alpha^{3}-8 \hbar^{2}+\hbar^{2} \alpha+\hbar \alpha+2 \hbar^{2} \alpha-4 \hbar^{3}\right. \\
\left.+\hbar \alpha^{3}-4 \hbar+\hbar^{3} \alpha^{3}\right) \mathrm{x}^{2}
\end{gathered}
$$

Then, the approximate solutions $\underline{\mathrm{F}}_{3}(\mathrm{x} ; \alpha)$ and $\overline{\mathrm{F}}_{3}(\mathrm{x} ; \alpha)$ are given by 


$$
\begin{aligned}
\underline{\mathrm{F}}_{3}(\mathrm{x} ; \alpha) & =\left(-\frac{56308742593741}{9223372036854775808} \hbar^{3} \alpha^{4}-\frac{563087425937441}{9223372036854775808} \hbar^{3} \alpha^{8}-\frac{1}{40950} \hbar^{3} \alpha^{7}-\frac{1}{27300} \hbar^{3} \alpha^{6}-\frac{1}{40950} \hbar^{3} \alpha^{5}\right) \mathrm{x}^{14} \\
& +\left(\frac{1}{1350} \hbar^{3} \alpha^{6}+\frac{1}{450} \hbar^{3} \alpha^{5}+\frac{1}{1350} \hbar^{3} \alpha^{3}+\frac{1}{450} \hbar^{3} \alpha^{4}\right) \mathrm{x}^{10}+\left(\frac{1}{5} \hbar^{2} \alpha^{3}+\frac{1}{10} \hbar^{2} \alpha^{2}+\frac{1}{5} \hbar \alpha^{3}+\frac{1}{10} \hbar^{2} \alpha^{4}\right. \\
& \left.+\frac{1}{10} \hbar \alpha^{2}+\frac{1}{10} \hbar \alpha^{4}\right) \mathrm{x}^{6}-\left(\hbar^{3} \alpha+\hbar^{3} \alpha^{2}+3 \hbar^{2} \alpha^{2}+3 \hbar \alpha^{2}+3 \hbar^{2} \alpha+3 \hbar \alpha\right) \mathrm{x}^{2}
\end{aligned}
$$

and

$$
\begin{aligned}
\overline{\mathrm{F}}_{3}(\mathrm{x} ; \alpha) & =\left(-\frac{32}{20475} \hbar^{3}-\frac{56308742593741}{9223372036854775808} \hbar^{3} \alpha^{12}-\frac{5433793660296007}{4611686018427387904} \hbar^{3} \alpha^{4}+\frac{32}{20475} \hbar^{3} \alpha-\frac{4}{6825} \hbar^{3} \alpha^{2}\right. \\
& \left.+\frac{2}{6825} \hbar^{3} \alpha^{7}-\frac{1}{1638} \hbar^{3} \alpha^{6}+\frac{2}{6825} \hbar^{3} \alpha^{5}-\frac{1}{27300} \hbar^{3} \alpha^{8}+\frac{2}{20475} \hbar^{3} \alpha^{9}-\frac{1}{40950} \hbar^{3} \alpha^{10}+\frac{34}{20475} \hbar^{3} \alpha^{3}\right) \mathrm{x}^{14} \\
& +\left(\frac{32}{675} \hbar^{3}-\frac{1}{1350} \hbar^{3} \alpha^{9}+\frac{2}{225} \hbar^{3} \alpha^{6}-\frac{49}{1350} \hbar^{3} \alpha^{3}-\frac{8}{225} \hbar^{3} \alpha+\frac{4}{225} \hbar^{3} \alpha^{4}-\frac{1}{450} \hbar^{3} \alpha^{5}-\frac{1}{450} \hbar^{3} \alpha^{7}\right. \\
& \left.+\frac{2}{225} \hbar^{3} \alpha^{2}\right) \mathrm{x}^{10}+\left(-\frac{4}{5} \hbar^{2} \alpha+\frac{1}{10} \hbar^{2} \alpha^{2}+\frac{1}{10} \hbar^{2} \alpha^{6}+\frac{1}{10} \hbar \alpha^{6}+\frac{8}{5} \hbar^{2}+\frac{8}{5} \hbar+\frac{1}{5} \hbar^{2} \alpha^{4}-\frac{4}{5} \hbar^{2} \alpha^{3}\right. \\
& \left.-\frac{4}{5} \hbar \alpha^{3}+\frac{1}{5} \hbar \alpha^{4}-\frac{4}{5} \hbar \alpha+\frac{1}{10} \hbar \alpha^{2}\right) \mathrm{x}^{6}+\left(3 \hbar^{2} \alpha^{3}-12 \hbar^{2}+\hbar^{3} \alpha+3 \hbar \alpha+3 \hbar^{2} \alpha-4 \hbar^{3}\right. \\
& \left.+3 \hbar \alpha^{3}-12 \hbar+\hbar^{3} \alpha^{3}\right) \mathrm{x}^{2}
\end{aligned}
$$

respectively.

To determine the valid region of the auxiliary parameter $\hbar$, we plot the $\hbar$-curves $\underline{F}_{3}(0.5 ; 0.5)$ and $\overline{\mathrm{F}}_{3}(0.5 ; 0.5)$ as shown in Figure 2. We can find that the region of $\hbar$ is the closed interval $[-1.4,-0.6]$. The error between exact and approximate solution is given in Table 2 .

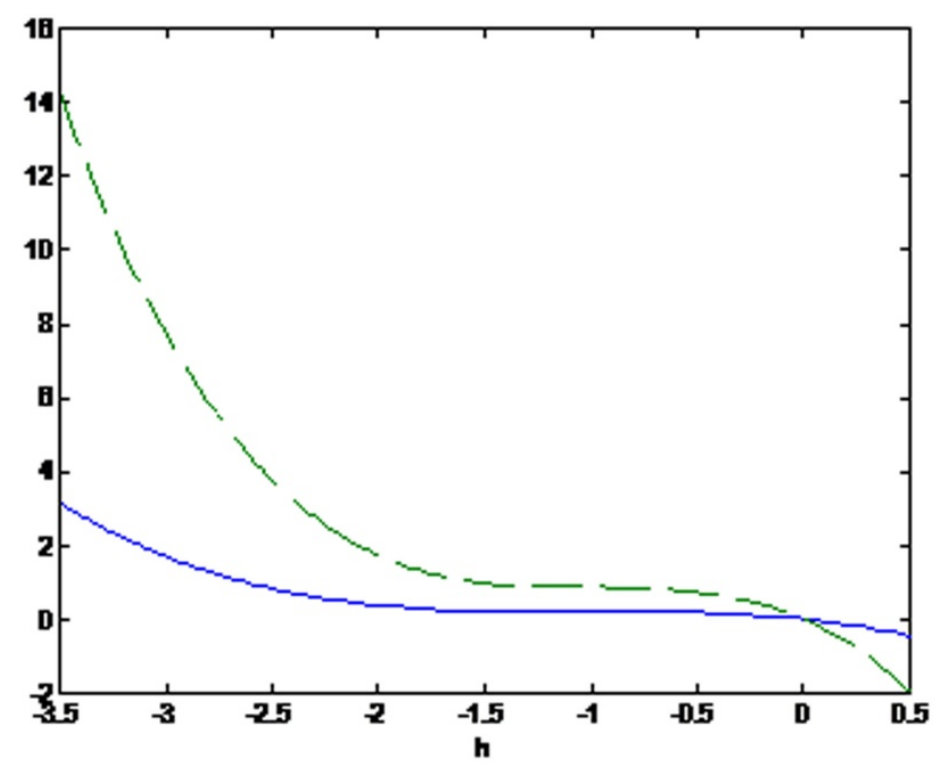

Figure 2. The $\hbar$-curve of $\underline{\mathrm{F}}_{3}(0.5 ; 0.5)$ and $\overline{\mathrm{F}}_{3}(0.5 ; 0.5)$, dashed dotted line represents $\underline{\mathrm{F}}_{3}(0.5 ; 0.5)$ and solid line represents $\overline{\mathrm{F}}_{3}(0.5 ; 0.5)$ 
Table 2. The error between exact and approximate solutions

\begin{tabular}{cccccccc}
\hline$x$ & $\hbar=-1.3$ & $\hbar=-1.2$ & $\hbar=-1.1$ & $\hbar=-1$ & $\hbar=-0.9$ & $\hbar=-0.8$ & $\hbar=-0.7$ \\
\hline 0 & 0 & 0 & 0 & 0 & 0 & 0 & 0 \\
0.1 & $1.1 \mathrm{e}-3$ & $3.2038 \mathrm{e}-4$ & $4.0176 \mathrm{e}-5$ & $4.7407 \mathrm{e}-12$ & $4.0144 \mathrm{e}-5$ & $3.2026 \mathrm{e}-4$ & $1.1 \mathrm{e}-3$ \\
0.2 & $4.4 \mathrm{e}-3$ & $1.3 \mathrm{e}-3$ & $1.7126 \mathrm{e}-4$ & $4.8543 \mathrm{e}-9$ & $1.6922 \mathrm{e}-4$ & $1.3 \mathrm{e}-3$ & $4.3 \mathrm{e}-3$ \\
0.3 & $1.0 \mathrm{e}-22$ & $3.2 \mathrm{e}-3$ & $4.8793 \mathrm{e}-4$ & $2.7986 \mathrm{e}-7$ & $4.6518 \mathrm{e}-4$ & $3.1 \mathrm{e}-3$ & $1.00 \mathrm{e}-2$ \\
0.4 & $1.9 \mathrm{e}-28$ & $6.7 \mathrm{e}-3$ & $1.4 \mathrm{e}-3$ & $4.9668 \mathrm{e}-6$ & $1.2 \mathrm{e}-3$ & $6.2 \mathrm{e}-3$ & $1.87 \mathrm{e}-2$ \\
0.5 & $3.6 \mathrm{e}-26$ & $1.39 \mathrm{e}-2$ & $3.7 \mathrm{e}-3$ & $4.6201 \mathrm{e}-5$ & $3.3 \mathrm{e}-3$ & $1.20 \mathrm{e}-2$ & $3.23 \mathrm{e}-2$ \\
0.6 & $6.7 \mathrm{e}-24$ & $2.89 \mathrm{e}-2$ & $9.3 \mathrm{e}-3$ & $2.8543 \mathrm{e}-4$ & $8.4 \mathrm{e}-3$ & $2.36 \mathrm{e}-2$ & $5.47 \mathrm{e}-2$ \\
0.7 & 0.1234 & $5.86 \mathrm{e}-2$ & $2.09 \mathrm{e}-2$ & $1.3 \mathrm{e}-3$ & $1.99 \mathrm{e}-2$ & $4.65 \mathrm{e}-2$ & $9.29 \mathrm{e}-2$ \\
0.8 & 0.2217 & 0.1125 & $4.20 \mathrm{e}-2$ & $5 \mathrm{e}-3$ & $4.40 \mathrm{e}-2$ & $9.02 \mathrm{e}-2$ & 0.1589 \\
0.9 & 0.3836 & 0.2020 & $7.52 \mathrm{e}-2$ & $1.62 \mathrm{e}-2$ & $9.16 \mathrm{e}-2$ & 0.1702 & 0.2716 \\
1 & 0.6313 & 0.3368 & 0.1190 & $4.58 \mathrm{e}-2$ & 0.1814 & 0.3115 & 0.4597 \\
\hline
\end{tabular}

It is worthy to point out that there are many applied problems that are modeled as integro-differential equations and can be solved by the current method such as; steady state condition of biological species living together, Maxwell equation in integro-differential form, chance to find time gap in dense traffic, etc.

\section{Conclusions}

In this paper we solved successfully the fuzzy Volterra integro-differential equations using Homotopy Analysis Method (HAM). It is apparently seen that HAM is very powerful and efficient technique in finding approximate solution for such equations. This method enjoys great freedom in choosing initial approximations, auxiliary linear operators and auxiliary functions. By means of this kind of freedom, a complicated problem can be transferred into an infinite number of simpler, linear subproblems that contain the auxiliary parameter $\hbar$ which provides a convenient way to adjust and control the convergence of the solution series, this represents the main advantage of this method.

\section{References}

Abbasbandy, S., Babolian, E., \& Ashtiani, M. (2009). Numerical solution of the generalized Zakharov equation by homotopy analysis method. Commune Nonlinear Sci. Num. Simulat., 14(12), 4114-4121. http://dx.doi.org/10.1016/j.cnsns.2009.03.001

Abbasbandy, S., Magyari, E., \& Shivanian, E. (2009). The homotopy analysis method for multiple solutions of nonlinear boundary value problems. Commun Nonlinear Sci. Num. Simulat., 14(9-10), 3530-3536. http://dx.doi.org/10.1016/j.cnsns.2009.02.008

Afroozi, G., Vahidi, A. J., \& Saeidy, M. (2010). Solving a class of two-dimensional linear and nonlinear integral equations by means of the Homotopy Analysis Method. International J. of nonlinear sciences, 9(2), 213-219.

Bataineh, A. S., Noorani, M. S. M., \& Hashim, I. (2008). Approximate analytical solutions of systems of PDEs by homotopy analysis method. Comp. and Math. With Appls., 55(12), 2913-2923. http://dx.doi.org/10.1016/j.camwa.2007.11.022

Bede, B., \& Gal, S. G. (2004).Almost periodic fuzzy-number-valued functions. Fuzzy Set Syst., 147(3), 385-403. http://dx.doi.org/10.1016/j.fss.2003.08.004

Bede, B., \& Gal, S. G. (2005). Generalizations of the differentiability of fuzzy number valued functions with applications to fuzzy differential equations. Fuzzy Set Syst., 151(3), 581-99.http://dx.doi.org/10.1016/j.fss.2004.08.001

Bede, B. (2008). Note on "Numerical solutions of fuzzy differential equations by predictor-corrector method". Information Sciences, 178(7), 1917-1922. http://dx.doi.org/10.1016/j.ins.2007.11.016

Chalco-Cano, Y., \& Roman-Flores, H. (2008). On new solutions of fuzzy differential equations. Chaos, Solitons and Fractals, 38(2), 112-119. http://dx.doi.org/10.1016/j.chaos.2006.10.043 
Chang, S. S. L., \& Zadeh, L. A. (1972). On fuzzy mapping and control. Trans. Systems, Man Cybernetics, SMC-2(1), 30-34. Retrieved from $\mathrm{http} / /$ ieeexplore.ieee.org $/ \mathrm{xpl} /$ login.jsp?tp=\&arnumber=5408553\&url=http\%3A\%2F\%2Fieeexplore.ieee.org \%2Fxpls\%2Fabs_all.jsp\%3Farnumber\%3D5408553

Dubois, D., \& Prade, H. (1982). Towards fuzzy differential calculus part 1. Fuzzy Sets and Systems, 8(1), 1-17. http://dx.doi.org/10.1016/0165-0114(82)90025-2

Friedman, M., Ma, M., \& Kandel, A. (1999). Numerical solutions of fuzzy differential and integral equations. Fuzzy sets and systems, 106(1), 35-48. http://dx.doi.org/10.1016/S0165-0114(98)00355-8

Ghanbari, M. (2010). Numerical Solution of Fuzzy linear Volterra integral equations of the second kind by Homotopy Analysis Method. Int. J. Indust. Math., 2(2), 73-87.

Goetschel, R., \& Voxman, W. (1983). Topological Properties of Fuzzy Numbers.Fuzzy Sets and Systems, 10(1-3), 87-99. http://dx.doi.org/10.1016/S0165-0114(83)80107-9

Goetschel, R., \& Voxman, W. (1986). Elementary fuzzy calculus.Fuzzy Sets and Systems, 18(1), 31-43. http://dx.doi.org/10.1016/0165-0114(86)90026-62

Kaleva, O. (1987). Fuzzy differential equations. Fuzzy Sets Systems, 24(2), 301-317. http://dx.doi.org/10.1016/0165-0114(87)90029-7

Liao, S. J. (2003). Beyond perturbation: introduction to the homotopy analysis method. Boca Raton: Chapman and Hall, CRC Press. http://dx.doi.org/10.1201/9780203491164

Liao, S. J. (2004). On the homotopy analysis method for nonlinear problem.Applied Mathematics and Computation, 147(2), 499-513. http://dx.doi.org/10.1016/S0096-3003(02)00790-7

Liao, S. J. (2009a). Series solution of nonlinear eigenvalue problems by means of the homotopy analysis method. Nonlinear Analysis: Real World Applications, 10(4), 2455-2470.

Liao, S. J. (2009b). Notes on the homotopy analysis method: Some definitions and theorems. Commun. Nonlinear Sci. Num. Simulat., 14(4), 983-997.

Liao, S. J., \& Tan, Y. (2007). A general approach to obtain series solutions of nonlinear differential equations. Stud. Appl. Math., 119(4), 297-355. http://dx.doi.org/10.1111/j.1467-9590.2007.00387.x

Mehrkanoon, S., Suleiman, M., \& Majid, Z. A. (2009). Block Method for Numerical Solution of Fuzzy Differential Equations. Int. Math. Forum, 4(46), 2269-2280.

Puri, M. L., \& Ralescu, D. (1983). Differential for fuzzy function. J. Math. Anal. Appl., 91(2), 552-558. http://dx.doi.org/10.1016/0022-247X(83)90169-5

Puri, M. L., \& Ralescu, D. (1986). Fuzzy random variables. J. Math. Anal. Appl., 114(2), 409-422. http://dx.doi.org/10.1016/0022-247X(86)90093-4 